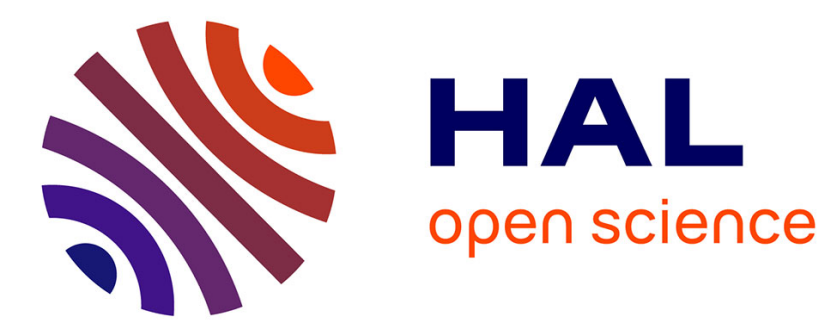

\title{
Boulder Fall Ejecta: Present Day Activity on Mars
}

S. Vijayan, H. Harish, K. B Kimi, S. Tuhi, K. Vigneshwaran, R. K Sinha, B. Sivaraman, Susan J. Conway, Anil Bhardwaj

\section{To cite this version:}

S. Vijayan, H. Harish, K. B Kimi, S. Tuhi, K. Vigneshwaran, et al.. Boulder Fall Ejecta: Present Day Activity on Mars. Geophysical Research Letters, 2022, 49 (1), pp.e2021GL096808. 10.1029/2021GL096808 . hal-03537861

\section{HAL Id: hal-03537861 https://hal.science/hal-03537861}

Submitted on 21 Jan 2022

HAL is a multi-disciplinary open access archive for the deposit and dissemination of scientific research documents, whether they are published or not. The documents may come from teaching and research institutions in France or abroad, or from public or private research centers.
L'archive ouverte pluridisciplinaire HAL, est destinée au dépôt et à la diffusion de documents scientifiques de niveau recherche, publiés ou non, émanant des établissements d'enseignement et de recherche français ou étrangers, des laboratoires publics ou privés. 
1

\section{Boulder Fall Ejecta: Present day activity on Mars}

\section{S. Vijayan ${ }^{1}$, Harish ${ }^{1}$, K.B. Kimi ${ }^{1}$, S. Tuhi ${ }^{2}$, K. Vigneshwaran ${ }^{3}$, R.K. Sinha ${ }^{1}$, S.J. Conway ${ }^{4}$, B. Sivaraman ${ }^{1}$, Anil Bhardwaj ${ }^{1}$}

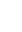

${ }^{1}$ Planetary Sciences Division, Physical Research Laboratory, Ahmedabad, India.

${ }^{2}$ College of Engineering, Anna university, Chennai, India.

${ }^{3}$ Department of Geology, Government college of Salem, Salem, India.

${ }^{4}$ CNRS, Laboratoire de Planétologie et Géodynamique UMR 6112, Université de Nantes, France.

Corresponding author: S Vijayan (vijayansiva@gmail.com)

\section{Key Points:}

- Comprehensive multitemporal HiRISE image analysis revealed all new boulder falls have ejecta along the track termed as Boulder fall ejecta

- $\quad 4500$ tracks with total integrated length of $~ 900 \mathrm{~km}$ formed in last few decades, 30\% BFE located in Cerberus Fossae suggests active region

- Some BFE fade within 2-4 Mars year and few persist >6 Mars year, evidence for slope streaks originating from BFE tracks support dry theory 


\begin{abstract}
Boulder falls are an archives of recent surface activity on Mars, however, determining how recently they fell remains elusive. Our multitemporal HiRISE image analysis shows that new tracks are characterized by a herringbone-like ejecta pattern at each boulder bounce that we call boulder fall ejecta(BFE). First systematic survey of BFE revealed $\sim 4500$ tracks whose total integrated track length is $\sim 900 \mathrm{~km}$. Our BFE longevity analysis reveals that these tracks likely formed in the last few decades. From few examples we also observed slope streaks originating from BFE tracks, providing additional evidence favoring a dry origin theory. BFE fade in as little as 2-4 Mars years, whereas, others can persist >6 Mars years suggesting differential weathering. Nearly $30 \%$ of BFE are observed in the Cerberus Fossae region, which suggests it is one of the most seismically active regions. Thus, BFE can be used to recognize very recent surface processes on planetary surfaces.
\end{abstract}

\title{
Plain Language Summary
}

High-resolution images of Mars have revealed numerous boulder falls on the surface. Here we systematically surveyed images from 2006 to 2020 to find recent boulder falls which have ejecta. The images of the same place taken at different times, revealed that all new boulder falls have ejecta which we call boulder fall ejecta (BFE). We searched all the images and found BFE present in large numbers suggesting recent falls are common and widespread. Our results revealed that on Mars it takes 2 to 4 Mars years (4 to 8 Earth years) for BFE to disappear, whereas on Earth BFE are rarely preserved. We found that the Cerberus Fossae region adjacent to NASA's InSight lander landing site hosts nearly $30 \%$ of BFE, suggesting it is a currently active regions on Mars.

\section{Introduction}

Boulder falls and their tracks are typically observed on Mars and the Moon using highresolution images (McEwen et al., 2007, Robinson et al., 2010). These falls indicate recent active mass wasting processes on these bodies. Such tracks are ubiquitous on steeply sloping terrain, and where particularly abundant have been used to infer active seismicity (Brown and Roberts, 2019; Taylor et al., 2013; Bickel et al., 2019), giving insight into endogenic processes. Repeat temporal imaging has shown that boulder falls are ongoing on Mars, as first observed by the Mars Global Surveyor - Mars Orbiter Camera (Malin and Edgett, 2001) images from the southern hemisphere and Valles Marineris (https://www.msss.com/mars_images/moc/2005/09/20/bouldertracks/). Subsequently, several studies have used repeat temporal imaging using HiRISE to identify and study recent boulder falls (Dundas et al., 2019; Grindrod et al., 2021; Dundas and Daubar, 2020). The boulder fall tracks are predominantly present on crater walls and floors, but can originate from any steeply sloping surface (Van der Bogert and Plescia, 2014; Kumar et al., 2019; Bickel et al., 2020; Sinha et al., 2020). Earlier studies have hypothesized that the main drivers for such falls are shallow marsquakes, wind/aeolian activity, impact-induced falls, and solar insolation (Roberts et al., 2012; Brown and Roberts, 2019; Kumar et al., 2019; Burleigh et al., 2012; Tesson et 
al., 2020). Yet, there is still ambiguity about the precise timing of boulder falls and the triggering and preconditioning factors of the falls.

In addition, boulder falls have been associated with the triggering of slope streaks (Chuang et al., 2007; Dundas, 2020). Slope streaks extend downslope on dust-covered sloped surfaces and have contrasting albedo (Sullivan et al., 2001). They generally originate from a small area or a point, located below knobs, spurs, crater rims, and individual boulders (Chuang et al., 2007; Dundas, 2020). Slope streaks appear as dark/light-toned features in comparison to the surrounding plains (Schorghofer et al., 2007). Darker streaks become lighter over time due to dust mantling (Sullivan et al., 2001; Aharonson et al., 2003; Motazedian et al., 2003; Schorghofer et al., 2007; Chuang et al., 2010). Some studies have noted a coincidence between the point where slope streaks originate and boulder fall tracks/bounces (Chuang et al., 2007; Yakovlev et al., 2020). Slope streaks are thought to result from a ground hugging flow of either dry (Sullivan et al., 2001; Chuand et al., 2007) or wet origin (Ferguson and Lucchitta, 1984; Bhardwaj et al., 2017).

In the initial reports of new temporal boulder fall tracks in MOC and HiRISE images, these tracks were found to have distinctive ejecta formed at each bounce. Here, we use the term boulder fall ejecta (BFE) to refer to a boulder bounce that repeatedly produces ejecta towards the downslope direction at every bounce point (Fig.1). Boulder fall tracks with BFE have never been systematically documented prior to this study. We performed a systematic survey of all impact craters in HiRISE images between 2006 and 2020 covering $\pm 35^{\circ}$ latitudes. For identification, mapping, and ejecta analysis see the methods described in the supplementary section for details. This study tackles the following open questions 1) do all new boulder falls cause BFE, 2) what is the spatial distribution of BFE on Mars, 3) what is the preservation potential or fading rate of BFE, and 4) the link between BFE tracks and slope streaks. This study yields important information to understand very recent boulder falls events, other associated present day activities and regolith mantling in planetary bodies.

\section{Boulder fall ejecta on Mars}

All new boulder falls mapped in this study (Fig.S1) using multi temporal HiRISE images have BFE and there are no tracks that appear without BFE (Fig.1; Figs.S2, S3) these are found at 33 different sites. The spatial spread of BFE varies from bounce-to-bounce and boulder-toboulder (Fig.1a). The ejecta are initiated at the point of contact between the boulder and the sloped surface, which forms a Herringbone shape (Oberbeck and Morrison, 1973). The ejecta appears v-shaped, with the spread pointing downslope (Fig.1b, c) and the spacing between each bounce is non-uniform (Fig.1d,e,f). We observed numerous tracks in close vicinity which had very different patterns of BFE spread (Fig.1). Another noticeable feature within or around numerous BFE tracks is the presence of smaller sub-tracks (Fig.1c,f). These sub-tracks are associated with larger main tracks and have a zigzag path with ejecta around them. They may be a result of boulders disintegrating during their fall or superposition on pre-existing boulder tracks. BFE tracks have a variety of lengths (maximum $\sim 2500 \mathrm{~m}$ ), and morphologies including straight, zigzag, and criss-crossing tracks with different bounce spacings, with subsequent variation in their size and ejecta spread (Fig.1).

The distribution of BFE tracks on Mars during the HiRISE observation period is shown in Fig.S1 and Supp Table T1. 32\% of BFE mapped in this study are identified in temporal 
HiRISE images (i.e. are not present in the first image, but are present in any of the later repeat coverage images). The formation of BFE seems independent of latitude, and the most recent BFE track formed in Mars Year 35 (Fig.S2). Our temporal observation of HiRISE images confirms that all new tracks are always associated with distinguishable ejecta along the track (Figs.S2,S3). Our finding that BFE are the result of boulder falls that occurred in the last decade(s) means that any BFE from the non-temporal images likely have an equivalent lifetime. So we searched for BFE and found that $68 \%$ are observed in non-temporal or from single HiRISE images (single image with no repeat coverage, or present in the first image of a temporal sequence) distributed over 70 sites. In total, we mapped $\sim 4530$ tracks, in which 1011 are tracks with temporal information $(\sim 180 \mathrm{~km})$ and 3519 are tracks seen only in individual images (covering $\sim 725 \mathrm{~km}$ ) (Supp Table 2).

We found that the ejecta mapped along several continuous large tracks reveals that the ejecta spread is greater during the initial bounces and decreases along the track in the downslope direction (Fig.S4). We mapped 53 long and continuous tracks located within different craters and this analysis revealed that nearly $55 \mathrm{~km}^{2}$ of surface dust had been modified during BFE formation. Taking this interpretation towards global scale boulder fall, suggests that a large amount of surface dust is disturbed during boulder falls. This suggests that the boulder falls on sloping surfaces will modify or expose the top of the regolith cover. We determined the slope over which the BFE are occurring based on available HiRISE digital terrain models. We found thatthe slopes where BFE formed are usually $<31^{\circ}$ (Fig.S4), which corresponds to common angle of repose and indicates movement beyond the originating slope (Howard, 1973; Kumar et al., 2016; Kumar et al., 2019).

Mars hosts numerous boulder tracks without ejecta (Roberts et al., 2012; Brown and Roberts, 2019; Kumar et al., 2019). Since all new boulder tracks in our survey have BFE, we infer that the absence of BFE suggests they were once present but have faded over time. In general, fading of boulder tracks over time is considered modification or evolution of the tracks (Dundas et al., 2019; Schaefer et al., 2019). To quantify the timescale of fading (Fig. S5)/ disappearance and preservation, we analyzed several BFE tracks with temporal images (Figs. 2 a-c;S6). Fig.2d shows the time intervals for the surveyed HiRISE temporal pairs and shows the fading rate for the BFE. For instance, BFE observed in a 2006 HiRISE image are still visible in 2020 images (Fig.S6c,d). This suggests that the preservation time for BFE is more than 6 Mars years. Similarly, we found another example where nearly 7 years post BFE formation, the ejecta along a boulder fall track is still visible (Fig.S3c,d). Fig.2a-c provides an example of a BFE which completely faded within $\sim 2$ to 4 Mars years. This study reveals that the first feature to disappear is the BFE during the evolution of boulder fall tracks. The fading of the BFE may occur via the same process as fresh dark slope streaks fade towards the background albedo overtime. However, the fading rate for the slope streaks is slower, i.e. 15 Mars years (Aharonson et al., 2003; Bergonio et al., 2013). The relatively fast fading rate of BFE is likely because of the smaller areal size of ejecta or because of differences in surface roughness or relief. Gradual or stochastic variation in dust deposition over various regions of Mars likely influences the fading or preservation time of BFE.

\section{Dark and Light toned BFE}


All the new tracks formed on Mars have BFE, and we observed that in $\sim 57 \%$ of cases they are darker and in $35 \%$ of cases are lighter than the surrounding terrain. In most locations, BFE are all darker or all lighter toned. In $7 \%$ of cases lighter and darker toned ejecta are found within the same host crater. This work provides the first observation of such lighter/darker toned BFE present in close proximity within crater (Fig.2a,b). Further, in two locations we observed contrasting ejecta forming in the same locality, (Fig.S8a,b). The lighter toned BFE are observed in the northern wall from the 2010 HiRISE image. Whereas to the immediate left side, darker toned BFE appear six year later in the 2016 HiRISE image. The geographic distribution of darker/lighter toned ejecta appears random, and no preference is observed, which suggests dust mantling is not homogeneous on Mars or tracks formed at different periods with different levels of dust.

\section{BFE and Slope streaks}

Two associations between BFE and slope steaks were observed. First BFE tracks formed over or crossed over slope streaks. For instance, we found an example crater (Fig.2g) where BFE tracks cross from the dark slope streak and enter into the adjacent lighter toned slope streaks. The BFE are dark toned when they are inside the dark slope streak and, when the BFE track enters the lighter slope streak, the BFE still have the same dark tone. We interpret that the lighter slope streaks are mantled by dust (Valantinas et al., 2021), which is then removed by the BFE. In another instance, a BFE track originated within a dark slope streak (Fig.2h,i) and extended to the adjacent surface, which is lighter in tone. The BFE did not change tone across the boundary. Thus, we infer that the boulder bounce is disturbing the top few centimeters of material to produce BFE, which then becomes dust mantled over time.

Secondly, in more than 20 locations, we find evidence that slope streaks originate from BFE tracks (Fig.3, Figs.S9-S11). A typical set of example is shown in Fig. 3, where the eastern crater wall hosts multiple BFE tracks, and from many of those tracks slope streaks originate from boulder bounce points. Many slope streaks originate from the middle of the track, where individual depressions with raised rims caused by bounces could act as a source for the dust avalanche. The slope streaks and BFE have similar albedo. In most cases, we observed that slope streaks formed from the BFE tracks broadened downslope, are darker than the surrounding terrain, and started at a point source, similarly to slope streaks in general. However, at multiple locations, we also observed shorter and narrower streaks than typical slope streaks (Fig.2d; Fig.S12). These streaks appear closely spaced and are formed at multiple places along the BFE track. In general, our observations suggest that the formation of slope streaks along the BFE tracks is contemporaneous and we have no temporal images showing that the slope streaks form after the boulder tracks have formed, but we acknowledge this might be possible even if we consider it unlikely.

Our observation that slope streaks form along fresh boulder tracks informs the debate on the origin for the slope streaks, i.e., whether they form by dry (Williams et al., 1991; Sullivan et al., 2001; Chuang et al., 2007) or wet (Ferguson and Lucchitta, 1984; Ferris et al., 2002; Bhardwaj et al., 2019) processes. Our observations show (Fig.3, Figs.S9-S11) that slope streaks originating from BFE tracks are randomly distributed with no latitudinal preference, and we find no evidence that the boulder falls themselves are related to wet processes. The BFE is the result of the excavation of the immediate subsurface material by each bounce of the boulder. As 
proposed previously (Williams, 1991; Chuang et al., 2007) the BFE related slope streaks are simply material mobilized downslope by dry processes.

\section{BFE formation}

The triggering mechanisms generally presumed for boulder falls on Mars are seismic, new impact craters, and/or wind/aeolian activity (Anderson et al., 1977; Roberts et al., 2012; Brown and Roberts, 2019; Dundas et al., 2019; Kumar et al., 2019; Malin and Edgett, 2001). For instance, a couple of BFE formed within a crater (Fig.4a) located on top of the Olympus Mons could be attributed most readily to wind/aeolian activity. This is because the scarps of the Olympus Mons caldera and other surrounding craters do not have any notable BFE, which might be expected if the trigger were seismic. Further, no nearby fresh impact could be found to explain the BFE.

Within several craters, we observed a large number of BFE tracks. For instance, one crater hosts a large number of BFE tracks (159 individual tracks) whose total integrated length is $\sim 71 \mathrm{~km}$ (Fig.4b). Among the mapped BFE, this particular crater has the most BFE in a single locality. Moreover, this crater hosts BFE with different sizes and spatial extent, which may be correlated to the boulder size, speed, or angle (Fig.4c). The difference in the spatial spread of the ejecta likely suggests the difference in the extent to which the surface is disturbed and possibly correlated to different boulder sizes. This location has only one HiRISE image, thus it cannot be determined whether the BFE formed coevally. Seismic events are more likely to cause regional increases in rockfall, so is less likely to be the principal factor here. To explain these highly localized concentrations of BFE, we infer that the source materials for the boulder falls are weaker than elsewhere or thermal cracking or several factors combined to trigger them.

Cerberus Fossae (CF) graben walls are known for their large number of boulder fall tracks without BFE and they have been hypothesized to be triggered by marsquakes (Roberts et al., 2012; Kumar et al., 2019). A previous study (Roberts et al., 2012) examined the size frequency distribution of the boulder tracks and another study by Dundas and Daubar (2020) reported a recent boulder fall in the CF graben. In addition to the observations of Dundas and Daubar (2020), we observed that the adjacent locations also host similar unreported BFE within the graben walls (Fig.S13). Only at three locations we observed BFE within the CF graben wall (Fig. S13). The BFE within the graben walls and within craters are similar. From our study, we found that the craters in and around the $\mathrm{CF}$ region host $\sim 30 \%$ of the BFE that we observed on Mars (Fig.S1). CF and the surrounding region craters mapped in this study host multiple BFE tracks with (Fig.S10) and without temporal information (Fig.3).

The InSight lander mission detected two of the largest marsquakes over the CF region (Giardini et al., 2020; Stahler et al., 2021; Kedar et al., 2021). In agreement, 30\% of the BFE identified in our survey are located around the Cerberus Fossae region and all of them are within craters. The $\mathrm{CF}$ and surrounding regions are the main hot spot for BFE (Fig.4d), and similar BFE on the CF graben walls provides compelling evidence for potentially currently active regions (Taylor et al., 2013). This study highlights that the seismic shaking originating from the CF has also likely affected the surrounding craters within the last few decades and may be related to the faults active in the recently detected events. Our new observations show that the Cerberus Fossae region hosts a large number of BFE which occurred within the last few decades and supports 
earlier studies (Roberts et al., 2012; Brown and Roberts, 2019) who found that it is one of the most seismically active region on Mars.

Zunil, Gratteri, and nine other unnamed craters host numerous BFE (Figs.S3c,d;S14), with and without temporal information within the same crater (Supp. Table 3). These observations (Fig.S7) suggest frequent boulder fall activity within the crater either at the same location or anywhere within the crater. All the BFE tracks mapped in this study formed side-byside with the older tracks without BFE, which suggests the boulder falls are frequent and occuring over decades on Mars. From our survey, we find rockfall triggered by wind, thearmal cracking and seismic shaking are reasonable triggers for the recent falls causing BFE. Our observations suggest that the present day occurrence of boulder falls is wide spread, and that they are happening more frequently (Fig.4d,e) than previously reported (Dundas and Daubar, 2020; Grindord et al., 2021).

\section{Implications for BFE on planetary bodies}

Evidence of boulder falls are very common on the Earth and on the Moon. On Earth, boulder falls do have ejecta during the fall, but they are quickly modified and removed, so cannot be systamatically studies. Recent boulder falls have been observed to produce ejecta (Fig.S15), but there have been no reports of it being preserved in the landscape. Hence, BFE on Mars presents a unique opportunity to study the dynamics of boulder-substrate interaction which could inform boulder fall studies essential to understanding the hazard they pose on Earth (Dorren 2003; Caviezel et al., 2021). On the Moon, there have been no reports of BFE along the tracks of boulder falls, despite the prevalence of fine-grained regolith at the lunar surface. Given the large number of boulder fall tracks on Moon, we thought it unlikely that BFE did not exist on the Moon and a short survey reveald that they do (Fig.S15) and are the subject of upcoming work. Thus, the presence of BFE on planetary bodies is an important piece of direct evidence for recent boulder falls within the last few decades and is a good indicator for geologically active regions.

\section{Acknowledgments}

The authors SV, H, KKB, RS, BS and AB thank the support provided by the Department of Space, Govt. of India for carrying out this work. We thank NASA and MRO team for collecting and processing all the HiRISE images used in the study. SV thanks Dr. Mangold, Dr. Alfred McEwen and Dr. C Dundas for initial discussions. SJC is supported for her HiRISE work by the French Space Agency, CNES. We thank the anonymous reviewers for their suggestions.

\section{Open Research}


All of the HiRISE images used in this study are available via https://www.uahirise.org/catalog or from NASA Planetary Data System. Supplementary Table provides the geographic locations, HiRISE id and details observed in each image.

\section{References}

Aharonson, O., Schorghofer, N. \& Gerstell, M. F. (2003), Slope streak formation and dust deposition rates on Mars. Journal of Geophysical Research: Planets 108.

Anderson, D. L. et al. (1977), Seismology on mars. Journal of Geophysical Research 82, 45244546.

Bergonio, J.R., Rottas, K.M. and Schorghofer, N., 2013. Properties of martian slope streak populations. Icarus, 225(1), pp.194-199.

Bhardwaj, A., Sam, L., Martín-Torres, F. J. \& Zorzano, M.-P. (2019), Are Slope Streaks Indicative of Global-Scale Aqueous Processes on Contemporary Mars? Reviews of Geophysics $57,48-77$.

Bhardwaj, A., Sam, L., Martín-Torres, F. J., Zorzano, M.-P. \& Fonseca, R. M. (2017), Martian slope streaks as plausible indicators of transient water activity. Scientific reports 7, 1-14.

Bickel, V. T. et al. (2019), Analysis of Lunar Boulder Tracks: Implications for Trafficability of Pyroclastic Deposits, Journal of Geophysical Research: Planets 124, 1296-1314.

Bickel, V. T., Aaron, J., Manconi, A., Loew, S. \& Mall, U. (2020), Impacts drive lunar rockfalls over billions of years, Nature communications 11, 1-7.

Brown, J. R. \& Roberts, G. P. (2019), Possible evidence for variation in magnitude for marsquakes from fallen boulder populations, Grjota Valles, Mars, Journal of Geophysical Research: Planets 124, 801-822.

Burleigh, K. J. et al. (2012), Impact airblast triggers dust avalanches on Mars. Icarus 217, 194201.

Caviezel, A., Ringenbach, A., Demmel, S.E. et al. (2021), The relevance of rock shape over mass- implications for rockfall hazard assessments. Nature Communications 12, 5546. 
Chuang, F. C., Beyer, R. A. \& Bridges, N. T. (2010), Modification of martian slope streaks by eolian processes. Icarus 205, 154-164.

Chuang, F. C., Beyer, R. A., McEwen, A. S. \& Thomson, B. J. (2007), HiRISE observations of slope streaks on Mars. Geophysical Research Letters 34.

Dorren, L. K. (2003), A review of rockfall mechanics and modelling approaches. Progress in Physical Geography 27, 69-87.

Dundas, C. M. \& Daubar, I. J. (2020), Observations of Mass Wasting Events in the Cerberus Fossae, Mars, 51st Lunar and Planetary Science Conference, held 16-20 March, 2020 at The Woodlands, Texas. LPI Contribution No. 2326, id.2404.

Dundas, C. M. (2020), Geomorphological evidence for a dry dust avalanche origin of slope streaks on Mars. Nature Geoscience 13, 473-476.

Dundas, C. M., Mellon, M. T., Conway, S. J. \& Gastineau, R. (2019), Active boulder movement at high Martian latitudes, Geophysical research letters 46, 5075-5082.

Ferguson, H. M. \& Lucchitta, B. K. (1984), Dark streaks on talus slopes, Mars. NASA Tech. Memo., NASA TM-86246 188-190.

Ferris, J. C., Dohm, J. M., Baker, V. R. \& Maddock III, T. (2002), Dark slope streaks on Mars: Are aqueous processes involved? Geophysical Research Letters 29, 128-1.

Giardini, D. et al. (2020), The seismicity of Mars. Nature Geoscience 13, 205-212.

Grindrod, P. M., Davis, J. M., Conway, S. J., \& de Haas, T. (2021). Active boulder falls in Terra Sirenum, Mars: Constraints on timing and causes. Geophysical Research Letters, 48, e2021GL094817.

Howard, K. A. (1973), Avalanche mode of motion: Implications from lunar examples. Science $180,1052-1055$.

Kedar, S. et al. (2021), Analyzing Low Frequency Seismic Events at Cerberus Fossae as Long Period Volcanic Quakes. Journal of Geophysical Research: Planets 126, e2020JE006518.

Kinnaur, Himachel Pradesh, India. Boulder fall https://www.youtube.com/watch?v=sFF2rhwdh$\underline{\text { w https://twitter.com/nagarkoti/status/1419248302864797696?lang=en }}$

Malin, M. C. \& Edgett, K. S. (2001), Mars global surveyor Mars orbiter camera: interplanetary cruise through primary mission, Journal of Geophysical Research: Planets 106, 23429-23570.

Mars Global Surveyor MOC2-1222 Release. https://www.msss.com/mars_images/moc/2005/09/20/bouldertracks/. 
McEwen, A. S. et al. (2007), Mars Reconnaissance Orbiter's High Resolution Imaging Science Experiment (HiRISE), Journal of Geophysical Research: Planets 112.

Motazedian, T. (2003), Currently flowing water on Mars. in Lunar and Planetary Science Conference 1840.

Oberbeck, V. R. \& Morrison, R. H. (1973), On the formation of the lunar herringbone pattern. in Lunar and Planetary Science Conference Proceedings vol. 4107.

Roberts, G. P., Matthews, B., Bristow, C., Guerrieri, L. \& Vetterlein, J. (2012), Possible evidence of paleomarsquakes from fallen boulder populations, Cerberus Fossae, Mars. Journal of Geophysical Research: Planets, 117.

Robinson, M. S. et al. (2010), Lunar reconnaissance orbiter camera (LROC) instrument overview, Space Science Reviews 150, 81-124.

Schaefer, E.I., McEwen, A.S. and Sutton, S.S., 2019. A case study of recurring slope lineae (RSL) at Tivat crater: Implications for RSL origins. Icarus, 317, pp.621-648.

Schorghofer, N., Aharonson, O., Gerstell, M. F. \& Tatsumi, L. (2007), Three decades of slope streak activity on Mars. Icarus 191, 132-140.

Senthil Kumar, P. et al. (2016), Recent shallow moonquake and impact-triggered boulder falls on the Moon: New insights from the Schrödinger basin. Journal of Geophysical Research: Planets $121,147-179$.

Senthil Kumar, P. et al. (2019), Recent seismicity in Valles Marineris, Mars: Insights from young faults, landslides, boulder falls and possible mud volcanoes, Earth and Planetary Science Letters 505, 51-64.

Sinha, R. K. et al. (2020), Boulder fall activity in the Jezero crater, Mars, Geophysical Research Letters 47, e2020GL090362.

Stähler, S. C. et al. (2021), Seismic detection of the martian core. Science 373, 443-4.

Sullivan, R., Thomas, P., Veverka, J., Malin, M. \& Edgett, K. S. (2001), Mass movement slope streaks imaged by the Mars Orbiter Camera. Journal of Geophysical Research: Planets 106, 23607-23633.

T. Heyer, H. Hiesinger, D. Reiss, G. Erkeling, H. Bernhardt, D. Luesebrink, R. Jaumann, The multi-temporal database of planetary image data (MUTED): A web-based tool for studying dynamic Mars, Planet. Space Sci., vol. 159, pp. 56-65, 2018.

Taylor, J., Teanby, N. A. \& Wookey, J. (2013), Estimates of seismic activity in the Cerberus Fossae region of Mars.,Journal of Geophysical Research: Planets 118, 2570-2581. 
Tesson, P.-A. et al. (2020), Evidence for thermal-stress-induced rockfalls on Mars impact crater slopes. Icarus 342, 113503.

Valantinas, A., Becerra, P., Pommerol, A., Tornabene, L.L., Affolter, L., Cremonese, G., Hauber, E., McEwen, A.S., Munaretto, G., Pajola, M. and Bowen, A.P., 2021. Color and multiangular observations of martian slope streaks. Planetary and Space Science, p.105373.

van der Bogert, C. H. \& Plescia, J. B. Boulder Track. in Encyclopedia of Planetary Landforms (eds. Hargitai, H. \& Kereszturi, Á.) 1-8 (Springer, 2014). doi:10.1007/978-1-4614-9213-9_19-1.

Williams, S. H. (1991), Dark talus streaks on Mars are similar to aeolian dark streaks. in Lunar and Planetary Science Conference vol. 22, Page 1509.

Yakovlev, V., Gorelik, S., Kreslavsky, M. \& Kovalenko, N. (2020), Deflecting Slope Streaks on Mars: Possible Formation Mechanism. 51st Lunar and Planetary Science Conference, held 16-20 March, 2020 at The Woodlands, Texas. LPI Contribution No. 2326, id.1230.

\section{Figures:}
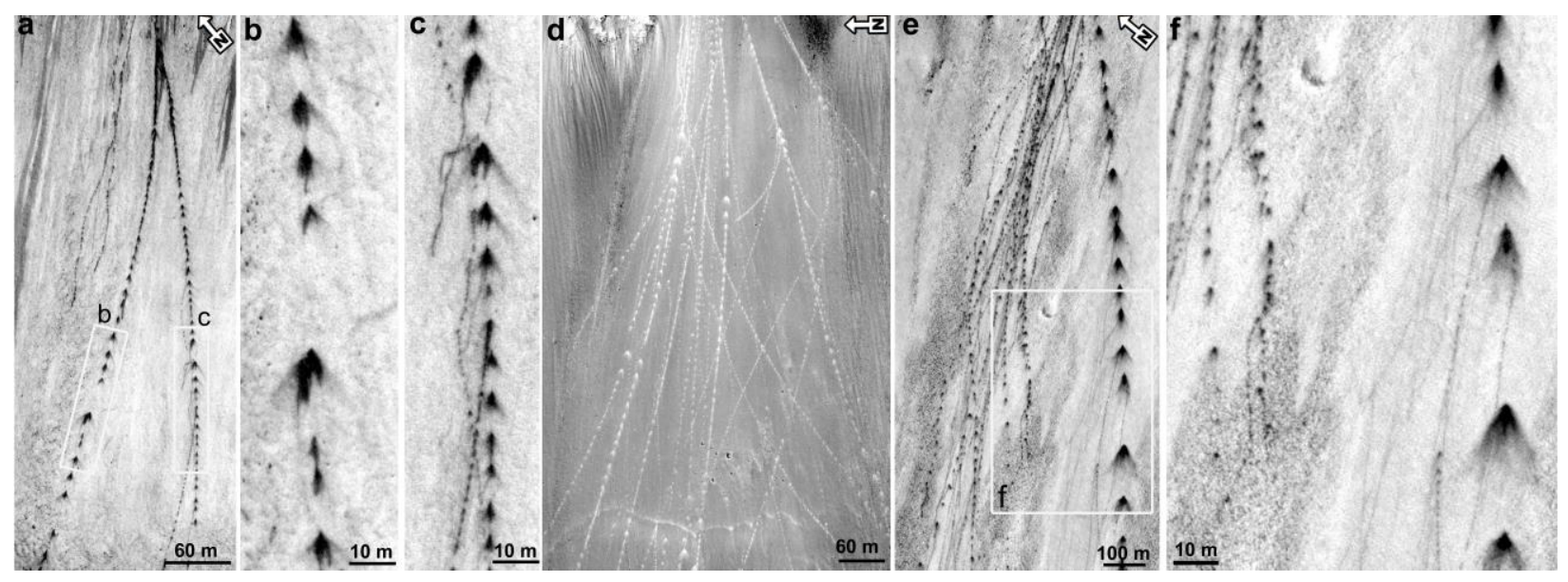

Figure 1. Formation of boulder track with ejecta. a-c), Boulder fall ejecta with a diverse spread of ejecta along the track (id: ESP_016396_2045). d), multiple boulder tracks with light toned ejecta (id: ESP_017975_1705). e), the ejecta spread along the track varies. f), spacing between the bounces and ejecta spatial spread varies and small sub-tracks from some boulder bounces (id: ESP_037287_2040). All images have an applied stretch to best highlight the BFE. 

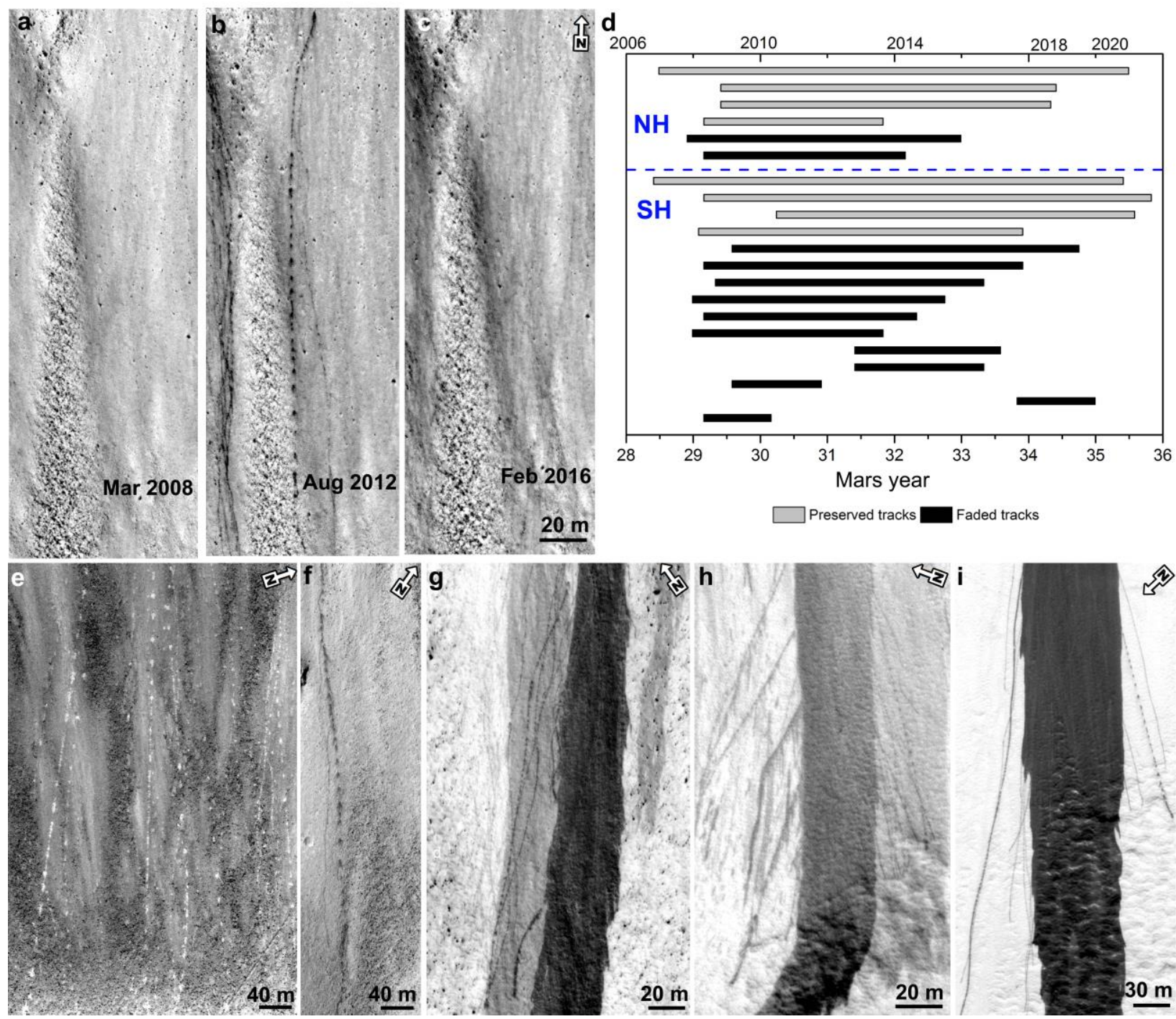

Figure 2. Example location for temporal BFE a) HiRISE image with no BFE track

434 (PSP_007735_1570), b) HiRISE image with BFE track (ESP_028345_1570) and c) HiRISE 435 image with BFE fully faded (ESP_044749_1570)). These 3 images in the same location provide 436 an ample evidence for the formation and fading of BFE. d) Time interval of HiRISE pairs used in 437 this study for fading and preserved BFEs. Faded tracks interval are varying from 1 Martian year to few Martian years, whereas the preserved tracks are having few Martian years. Evidence for dark and light toned ejecta. e,f) locations are within a crater and nearby region, but the ejecta 
440 formed by the boulder are darker or lighter in tone (id: ESP_023090_1690). g), BFE track 441 passing from dark streak to adjacent light streak, but the emplaced ejecta is darker, possibly 442 indicating different dust mantling (id: ESP_016396_2045). h), another example BFE track from 443 dark streak region enters light toned surface and light toned streak, in both the ejecta are dark 444 toned. Small, downslope-oriented narrow streaks emanate from each bounce point (id: 445 ESP_016396_2045). i), BFE track passing from within a slope streak to the adjacent light toned 446 surface, whereas the ejecta is dark toned (id: ESP_025840_1695). 


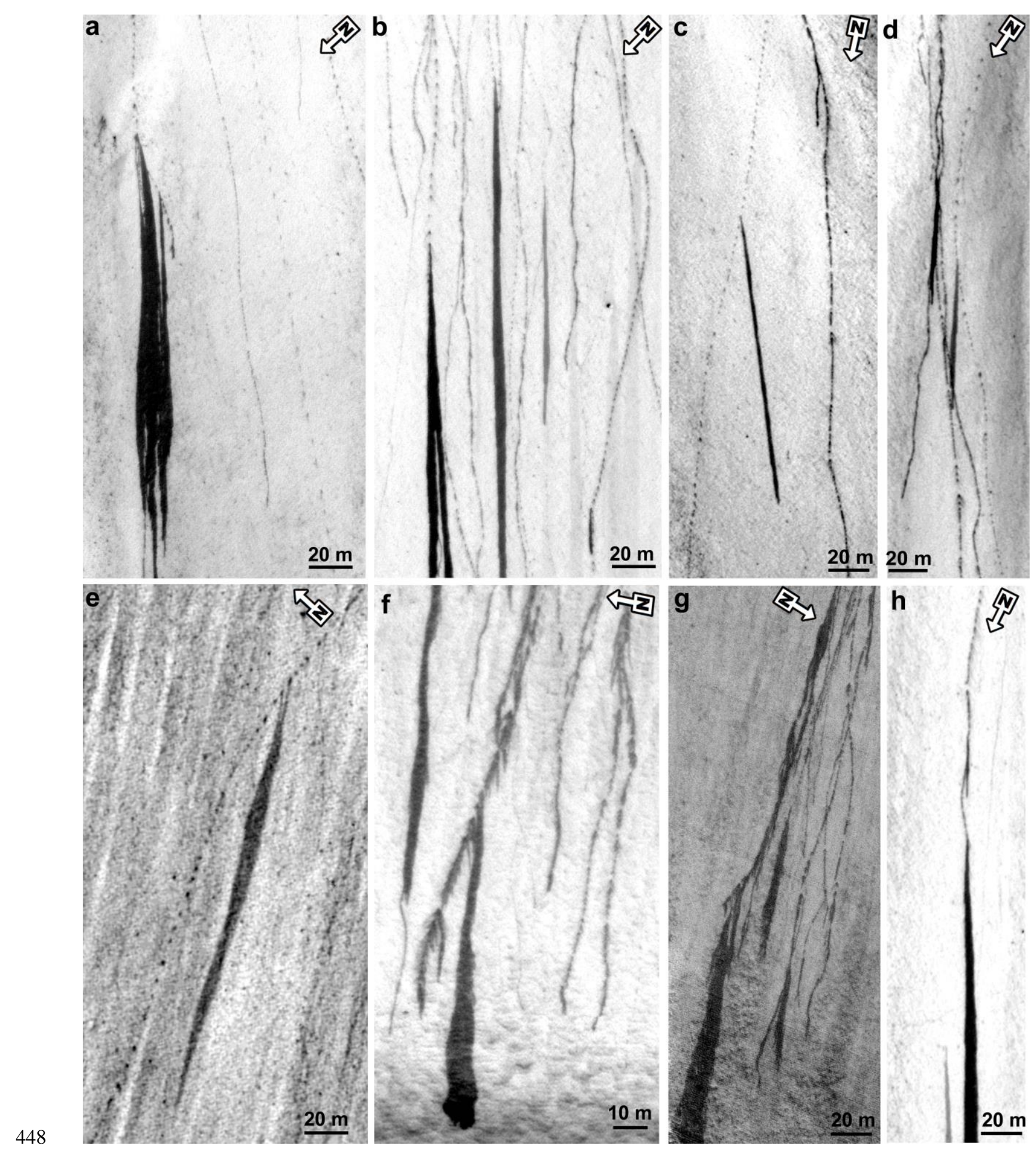

449 Figure 3. Comprehensive evidence for streaks originating from BFE track. a-e), slope streaks 450 seeming to form from the BFE track. They have widths and extent typical of other slope streaks.

451 Many slope streaks originate from the middle of track and each boulder bounce. The small raised 
452 surface by the boulder acts as a point source for slope streak formation (id: ESP_037287_2040).

453 f), slope streak emanating from the middle of the BFE track (id: ESP_016396_2045). g), slope

454 streak emanating from the BFE track within Zunil crater's western wall (id: PSP_002397_1880).

h), a distinguishable slope streak from the BFE track (id: ESP_037287_2040). The fresh boulder
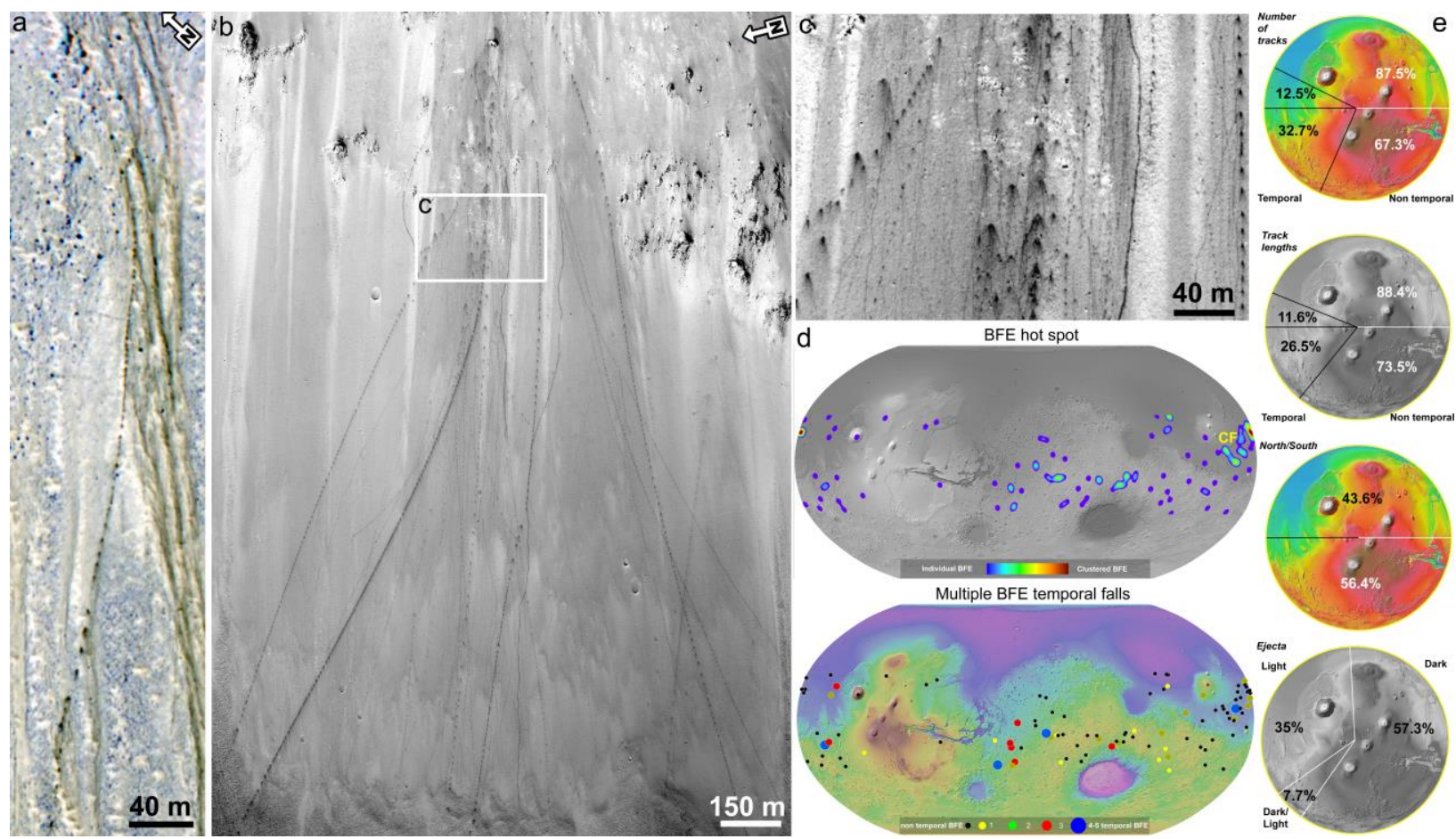

Figure 4. Global BFE distribution and formation. a), BFE formed within a crater located on top of Olympus Mons (id: PSP_005533_1975_Color). b), a Large number of BFE tracks formed within a crater whose total integrated track length is $~ 71 \mathrm{~km}$ (id: ESP_026651_1525). c), BFE tracks with very diverse ejecta spread, bounce distance, and sizes. d), hot spot map shows that

464 Cerberus Fossae and the surrounding region contains a large number of BFE indicating presentday activity. The southern highlands also host some hot spots for boulder falls. Locations of BFE 
466 where multiple falls occurred within the same crater (CF: Cerberus Fossae region). e), the 467 statistical distribution of the number of tracks, track lengths, spatial spread of tracks and ejecta. 